\title{
APLICAÇÃO DE FEIXES DE INTERVENÇÃO NUMA UNIDADE DE TERAPIA INTENSIVA PORTUGUESA
}

\author{
APPLICATION OF INTERVENTION BUNDLES IN A \\ PORTUGUESE INTENSIVE CARE UNIT
}

\section{APLICACIÓN DE VIGAS DE INTERVENCIÓN EN UNA UNIDAD PORTUGUESA DE CUIDADOS INTENSIVOS}

\author{
Ana Gertrudes Felgueiras Machado ${ }^{1}$ \\ Amâncio António de Sousa Carvalho ${ }^{2}$ \\ Ana Paula Morais Carvalho Macedo ${ }^{3}$
}

Como citar este artigo: Machado AGF, Carvalho AAS, Macedo APMC. Aplicação de Feixes de Intervenção numa unidade de terapia intensiva portuguesa. Rev baiana enferm. 2021;35:e-43809.

Objetivo: identificar a associação entre a aplicação de Feixes de Intervenção e características sociodemográficas e profissionais de enfermeiros em uma unidade de terapia intensiva portuguesa. Método: estudo descritivo e transversal, com amostra de 44 enfermeiros de uma Unidade de Terapia Intensiva Portuguesa. Na coleta de dados, aplicou-se questionário de maio a junho de 2019. Tratamento incluiu estatística descritiva e inferencial. Resultados: a maioria da amostra não executava auditorias $(86,4 \%)$ quanto às infeções em estudo. A pontuação do Feixe de Intervenção da Infeção do Trato Urinário e da Pneumonia Associada à Intubação diferiu significativamente entre os sexos ( T de Student: p<0,047 e p<0,037), bem como entre o exercício profissional total e tempo de exercício na Unidade de Terapia Intensiva, respetivamente (T de Student: $\mathrm{p}<0,001$ e p<0,026). Conclusão: a aplicação dos Feixes de Intervenção associou-se às características sociodemográficas e ao tempo de exercício profissional de enfermeiros de unidade de terapia intensiva portuguesa.

Descritores: Infecções. Controle de Infecções. Auditoria de Enfermagem. Cuidados Críticos. Enfermeiras e Enfermeiros.

Objective: to identify the association between the application of Intervention Bundles and sociodemographic and professional characteristics of nurses in a Portuguese intensive care unit. Method: descriptive and cross-sectional study, with a sample of 44 nurses from a Portuguese Intensive Care Unit. A questionnaire was applied from May to June 2019 in the data collection. Treatment included descriptive and inferential statistics. Results: the majority of the sample did not perform audits (86.4\%) regarding the infections under study. The score of the Intervention Bundle of Urinary Tract Infection and Pneumonia Associated with Intubation differed significantly between the genders (Student's T: $p<0.047$ and $p<0.037$ ), as well as between the total professional exercise and exercise time in the Intensive Care Unit, respectively (Student's T: $p<0.001$ and $p<0.026$ ). Conclusion: the application of the Intervention Bundles was associated with sociodemographic characteristics and time of professional practice of nurses from a Portuguese intensive care unit.

Descriptors: Infections. Infection Control. Nursing Audit. Critical Care. Nurses.

\footnotetext{
Enfermeira. Mestre em Enfermagem da Pessoa em Situação Crítica. Enfermeira do Centro Hospitalar do Tâmega e Sousa, Serviço de Medicina. Penafiel, Portugal. http://orcid/0000-0002-8525-7| 5X.

2 Enfermeiro. Mestre em Educação. Doutor em Estudos da Criança. Professor Adjunto da Escola Superior de Saúde, Universidade de Trás-os-Montes e Alto Douro. Vila Real, Portugal. amanciocarv@hotmail.com. http://orcid/0000-0002-I573-53।2.

3 Enfermeira. Mestre e Doutora em Educação. Health Sciences Research Unit: Nursing (UICISA: E), Nursing School of Coimbra (ESEnfC), Portugal. Professora Coordenadora na Escola Superior de Enfermagem da Universidade do Minho. Braga, Portugal. http://orcid/0000-0002-1064-3523.
} 
Objetivo: identificar la asociación entre la aplicación de Vigas de Intervención y las características sociodemográficas $y$ los profesionales de enfermería en una unidad de cuidados intensivos portuguesa. Método: estudio descriptivo y transversal, con una muestra de 44 enfermeras de una Unidad de Cuidados Intensivos portuguesa. Se aplicó un cuestionario de mayo a junio de 2019 en la recolección de datos. El tratamiento incluyó estadísticas descriptivas e inferenciales. Resultados: la mayoría de la muestra no realizó auditorías (86,4\%) respecto a las infecciones en estudio. La puntuación del Vigas de Intervención de Infección urinaria y Neumonía Asociada a Intubación difirió significativamente entre los sexos ( $T$ de Student: $p<0,047$ y $p<0,037)$, asi como entre el ejercicio profesional total y el tiempo de ejercicio en la Unidad de Cuidados Intensivos, respectivamente ( $T$ de Student: $p<0,001$ y $p<0,026$ ). Conclusión: la aplicación de los Vigas de Intervención se asoció con características sociodemográficas y tiempo de práctica profesional de las enfermeras de una unidad de cuidados intensivos portuguesa.

Descriptores: Infecciones. Control de Infecciones. Auditoría de enfermería. Cuidados Críticos. Enfermeras y Enfermeros.

\section{Introdução}

A prestação de cuidados de saúde em meio hospitalar pode dar origem à transmissão de Infeções Associadas a Cuidados de Saúde (IACS). Se, por um lado, prolonga-se a sobrevida de doentes, por outro verifica-se que os mesmos doentes tornam-se vulneráveis às múltiplas infeções que podem adquirir nos locais onde ocorre a prestação de cuidados, sobretudo devido ao recurso a procedimentos mais invasivos, à terapêutica antibiótica agressiva ou imunossupressora e às internações subsequentes, como nas Unidades de Terapia Intensiva (UTI).

No último século assistiu-se a um aumento significativo da longevidade, mas nem sempre o prolongamento da vida ocorre com a qualidade desejável. Os períodos passados em internação são caraterizados por uma diminuição das defesas do organismo, isto é, os mecanismos de defesa são anulados pelas técnicas de diagnóstico, monitorização ou terapêutica que os deprimem ou ultrapassam, gerando-se condições para a ocorrência de infeções ${ }^{(1)}$.

As IACS podem definir-se como infeções adquiridas após a admissão do paciente numa unidade de saúde. Manifestam-se durante a internação ou após a alta e relacionam-se com esses dois procedimentos. Em estudo realizado no Brasil, 28,3\% dos pacientes apresentaram IACS durante o primeiro mês do pós-operatório ${ }^{(2)}$. Para além do aumento substancial da morbilidade dos doentes internados em contexto hospitalar, a IACS constitui-se como uma das maiores causas de morte em todo o mundo.
O uso de cateteres vasculares centrais (CVC) é um dos fatores de risco mais conhecidos relacionados com as IACS. Uma infeção da corrente sanguínea relacionada ao CVC ocorre quando o microrganismo presente no local de inserção invade a corrente sanguínea, desencadeando uma bacteriemia, que pode provocar uma sépsis, ao não ser tratada adequadamente ${ }^{(3)}$. Esta contaminação representa a principal causa de infeção em UTI. Os autores desse estudo referem que, na Europa, verificam-se 13,3 infeções por 1.000 cateteres/dia, acrescentando que essa infeção está associada ao aumento do tempo de internação entre 10 a 20 dias e a um custo aproximado de US\$ 30 mil por paciente ${ }^{(4)}$. Outro estudo menciona também o aumento dos custos na assistência à saúde ${ }^{(5)}$ e outro reporta que, nos Estados Unidos da América, o custo para um episódio de infeção variou de US\$ 24.090 até US\$ 34.544 e na Europa entre 16.814 EUR e 29.909 EUR ${ }^{(6)}$.

Por sua vez, a Infeção do Trato Urinário (ITU) diz respeito à presença e multiplicação de bactérias no trato urinário, provocando danos nos tecidos, desde a uretra até os rins. Essas infeções, frequentes em doentes hospitalizados, são comuns em todo o mundo e estima-se que 150 milhões de pessoas sejam afetadas por ano ${ }^{(7)}$. Dentre as IACS 40\% são ITU, que apresentam baixa mortalidade ${ }^{(8)}$. Segundo estudo desenvolvido no Bahrain, as ITU são as infeções hospitalares mais comuns em todo o mundo. Estão associadas ao aumento da mortalidade, morbilidade, duração do internação e custos de saúde, sendo 
o cateterismo urinário o fator de risco mais importante para o seu desenvolvimento ${ }^{(9)}$.

No dizer do Programa de Prevenção e Controlo de Infeções e de Resistência aos Antimicrobianos ${ }^{(10)}$, embora a prevalência da Infeção associada à CVC $(57,1 \%)$ e à Pneumonia Associada à Intubação (PAI) (36,6\%), em UTI de adultos, entre os anos 2008-2016, tenha vindo a baixar e se tenha verificado também uma redução da prevalência das IACS em meio hospitalar para 7,8\%, estes percentuais continuam elevados. Quanto à PAI, um estudo realizado em Portugal refere que um paciente intubado tem um risco três a dez vezes superior de desenvolver pneumonia comparada a um paciente não intubado. A PAI pode atingir 7 a 30\% das pessoas com ventilação mecânica invasiva ${ }^{(11)}$.

Segundo uma organização europeia ${ }^{(12)}, 8,3 \%$ dos doentes que permaneceram internados na UTI por mais de dois dias apresentaram infeções, tais como a pneumonia, a infeção do CVC e a ITU. Dos doentes internados por mais de dois dias na UTI, 6\% apresentaram: pneumonia, sendo 98\% dos casos relacionados com a intubação (PAI); 4\% apresentaram infeção da corrente sanguínea, sendo $37 \%$ dos casos relacionados com o CVC; e 2\% apresentaram ITU, sendo 98\% dos casos relacionados com a presença de cateter urinário.

A este respeito, autores ${ }^{(13)}$ realçam que as infeções mais frequentes são a PAI, a do CVC e a ITU, além de indicarem Feixes de Intervenção (FI) a serem implementados nas UTI, para prevenção de cada uma dessas infeções. Outros autores $^{(14)}$ reforçam esse entendimento, ao referirem que as IACS têm impacto na vida dos doentes, causando hospitalizações prolongadas, aumento da morbilidade e da mortalidade, das quais resultam um aumento significativo dos custos, tanto para os sistemas de saúde como para a sociedade. Acrescentam ainda que os FI foram implementados para melhoria nas práticas clínicas e para prevenção da infeção.

Ao longo do tempo, tem surgido um número crescente de intervenções baseadas em conjuntos de medidas dirigidas a determinado objetivo (bundles ou FI), aplicadas de forma coordenada e monitorizadas sistematicamente com recurso a listas de verificação (checklists), implicando a formação dos profissionais envolvidos. Esta abordagem tem tido resultados positivos, traduzindo-se na diminuição das infeções. Tratando-se de um conjunto coeso de medidas, elas têm de ser implementadas em conjunto, para o sucesso ser atingido, devendo ser objeto de auditoria.

A Direção-Geral da Saúde ${ }^{(1)}$ também recomenda que, na implementação dos FI, haja uma especial atenção ao caráter multidisciplinar, agregador e motivacional da equipe de trabalho. Esta equipe deverá ser capaz de monitorizar os resultados da aplicação dos FI, nomeadamente a adesão dos profissionais à aplicação das medidas dos FI, tendo em vista a melhoria dos indicadores. Este tipo de abordagem tem como objetivo a diminuição de incidência das infeções-alvo em pacientes internados. De acordo com a organização de saúde portuguesa ${ }^{(15)}$, a auditoria é um processo sistemático, independente e documentado para obter evidência objetiva e respetiva avaliação objetiva, com vista a determinar em que medida os critérios da auditoria são cumpridos, podendo contribuir para a aplicação desses FI.

Dado o caso do impacto que as taxas de IACS têm nos serviços de terapia intensiva e nas organizações de saúde em geral, surge o interesse em estudar os fatores que possam estar associados à aplicação de FI por enfermeiros, em contexto de uma UTI, no que diz respeito à prevenção de infeção. Nesse sentido, o objetivo deste estudo é identificar a associação entre a aplicação de FI e características sociodemográficas e profissionais de enfermeiros em uma UTI portuguesa.

\section{Método}

Trata-se de um estudo descritivo e transversal $^{(16)}$. A população alvo deste estudo era constituída por cerca de 58 enfermeiros, que exerciam funções nas UTI de um Centro Hospitalar da zona Norte de Portugal em três serviços: Unidade de Cuidados Intensivos Polivalente do Serviço de Urgência (UCIPSU) - 
11 enfermeiros; Unidade de Cuidados Intensivos Polivalente (UCIP) - 17 enfermeiros; e Unidade de Cuidados Intensivos de Cardiologia (UCIC) - 30 enfermeiros. Estabeleceu-se como critérios de inclusão: enfermeiros que exerciam a sua atividade profissional nas UTI do Centro Hospitalar da Região Norte de Portugal; enfermeiros que se encontravam na área da prestação de cuidados. Definiu-se como critérios de exclusão: enfermeiros que estivessem ausentes do serviço durante o período de recolha de dados; enfermeiros que estivessem de baixa médica durante o período de recolha de dados. A amostra ficou composta por 44 enfermeiros (cerca de 75,9\% da população) que se encontravam presentes no momento de recolha de dados. Destes, 11 pertenciam à UCIPSU (100\% da população), 17 à UCIP (100\% da população) e 16 à UCIC $(53,3 \%)$ da população.

A recolha de dados foi feita por meio da aplicação de um questionário de autopreenchimento constituído por cinco partes: a primeira parte destinada à caraterização sociodemográfica; a segunda, para caraterização da experiência e da formação profissional; a terceira, por questões respeitantes aos procedimentos de prevenção; a quarta, constituída por escalas relativas aos três FI construídas pelos investigadores; e a quinta e última, por questões relativas à realização de auditorias. Após ter sido concedida a autorização, por parte da administração do Centro Hospitalar da Região Norte de Portugal, para a realização do estudo (Parecer № 15/2019, de 1 de abril de 2019), foi agendada uma data para colheita de informação, que se realizou entre maio e junho de 2019. Para a aplicação do questionário, solicitou-se a colaboração das Enfermeiras-Chefes, tendo sido informadas acerca dos objetivos do estudo e procedimentos envolvidos. Elas assumiram a tarefa de informar a sua equipe de enfermagem acerca do estudo e solicitaram que respondessem ao questionário. Foram assegurados o anonimato e a confidencialidade da informação e a participação voluntária. Foi entregue um questionário e um envelope (para ser utilizado na devolução) aos enfermeiros que voluntariamente aceitaram participar no estudo. As Enfermeiras-Chefes de cada um dos serviços reuniram os envelopes fechados e respetivos questionários e, cerca de 30 dias após a entrega, os devolveram aos investigadores.

Para análise e tratamento de dados foi construída uma base de dados no software informático Statistical Package for the Social Sciences (SPSS), versão 25.0. De seguida, recorreu-se à estatística descritiva com cálculo das frequências absolutas e relativas para todas as variáveis e das medidas de tendência central (Média) e de dispersão (Desvio-padrão) para as variáveis de nível de medição rácio. Foram aplicados testes paramétricos (t de Student e ANOVA) no cruzamento entre a pontuação das escalas dos FI e os fatores analisados e, em alternativa, quando não estavam garantidos os pressupostos para a sua utilização, os testes não paramétricos Mann-Whitney e Kruskal-Wallis. Foi utilizado o teste de Shapiro-Wilk para avaliar a normalidade $\mathrm{da}$ distribuição das variáveis $(\mathrm{p} \geq 0,05)$. O nível de significância tido em conta foi de $5 \%{ }^{(17)}$.

\section{Resultados}

A maioria dos 44 profissionais de enfermagem da amostra era do sexo feminino, pertencia ao grupo etário dos 38-50 anos, possuía o estado civil de casado e detinha a licenciatura como habilitação académica (Tabela 1). A média da idade foi de 38,93 55,470 anos; o mínimo, os 26 anos, e o máximo, 50 anos (dados que não constam da tabela).

Tabela 1 - Caracterização sociodemográfica da amostra. Penafiel, Portugal - 2019. (N=44) (continua)

Variáveis
Sexo

Masculino

Feminino
Frequência absoluta Frequência relativa (\%) 
Tabela 1 - Caracterização sociodemográfica da amostra. Penafiel, Portugal - 2019. (N=44) (conclusão)

\begin{tabular}{l|c|c}
\hline Variáveis & Frequência absoluta & Frequência relativa (\%) \\
\hline Grupo etário & & \\
$26-37$ anos & 18 & 40,9 \\
$38-50$ anos & 25 & 56,8 \\
Não respondente & 1 & 2,3 \\
Estado civil & & 70,5 \\
$\quad$ Casado & 31 & 22,7 \\
Solteiro/a & 10 & 6,8 \\
Divorciado/a & 3 & \\
Habilitações Académicas & & 95,5 \\
Licenciatura & 42 & 4,5 \\
Mestrado & 2 & \\
\hline
\end{tabular}

Fonte: Elaboração própria.

A amostra de enfermeiros deste estudo enquadrava-se predominantemente no intervalo dos 16-30 anos de experiência profissional e possuía entre 9-20 anos de tempo de exercício profissional na UTI; o maior grupo de enfermeiros possuía uma especialidade e, destes, a maioria era na área de especialidade de médico-cirúrgica (Tabela 2).

Tabela 2 - Caracterização da experiência e formação profissional dos enfermeiros da amostra. Penafiel, Portugal - 2019. ( $N=44)$

\begin{tabular}{l|cc}
\hline Variáveis & Frequência absoluta & Frequência relativa (\%) \\
\hline Tempo de exercício profissional & & 38,6 \\
3-15 anos & 27 & 61,4 \\
16-30 anos & & \\
Tempo de Exercício na Unidade de & 18 & 40,9 \\
Cuidados Intensivos & 26 & 59,1 \\
1-8 anos & & \\
9-20 anos & & 13,6 \\
Formação & 6 & 25,0 \\
Pós-Graduação & 11 & 20,5 \\
Especialidade & 9 & 40,9 \\
As duas formações & 18 & 10,0 \\
Não respondente & & 50,0 \\
Especialidade (N=20) & 2 & 35,0 \\
Comunitária & 10 & 5,0 \\
Médico-Cirúrgica & 7 & \\
Reabilitação & 1 & \\
Saúde Materna e Obstetra & & \\
\hline
\end{tabular}

Fonte: Elaboração própria.

Quanto aos procedimentos de prevenção, a maioria dos enfermeiros utilizou luvas na prestação de cuidados $(61,4 \%)$ e considerou que o uso de luvas previne a infeção do doente e do profissional de saúde (79,5\%). A totalidade da amostra considerou que a higienização adequada das mãos previne as infeções (100\%) e a maioria assinalou fazer a higiene das mãos com SABA ou água e sabão (88,6\%).

Em relação à realização das auditorias relativas à aplicação dos FI, verificou-se, no caso da ITU,
CVC e PAI, respetivamente, que a maioria dos enfermeiros assinalou que o serviço era sujeito a auditoria (94,7\%, 100\%, 52,6\%); relativamente à frequência em que era executada a monitorização da adesão dos FI, a maioria assinalou que era executada às vezes (57,9\%), e as auditorias eram executadas em suporte informático $(73,1 \%)$.

No que concerne à associação entre a aplicação dos FI e as variáveis sociodemográficas, a média da pontuação do FI da ITU não diferiu significativamente entre os enfermeiros com 
diferentes grupos etários. A pontuação deste FI divergiu entre os enfermeiros dos dois sexos, tendo os enfermeiros do sexo feminino obtido uma média mais elevada, ao assinalarem ter melhores procedimentos nesse âmbito.

A média da pontuação do FI do CVC não foi dissente entre os enfermeiros de diferentes sexos e grupos etários.
A pontuação do FI da PAI distinguiu-se significativamente entre os enfermeiros dos dois sexos e dos grupos etários. Os enfermeiros do sexo masculino obtiveram uma média mais elevada, o que significa que assinalaram ter melhores práticas nesse FI, bem como os enfermeiros mais velhos, que obtiveram uma média mais elevada do que os mais novos (Tabela 3).

Tabela 3 - Associação entre a aplicação dos Feixes de Intervenção e as variáveis sociodemográficas. Penafiel, Portugal - 2019. (N=44)

\begin{tabular}{|c|c|c|c|c|c|c|}
\hline Variáveis & Categorias & $\mathbf{n}$ & $\begin{array}{c}\text { Média } \pm \text { desvio } \\
\text { padrão }\end{array}$ & $\begin{array}{c}\text { Valor do } \\
\text { teste }\end{array}$ & $\begin{array}{l}\text { Graus de } \\
\text { liberdade }\end{array}$ & $\begin{array}{l}\text { Teste t de } \\
\text { Student p }\end{array}$ \\
\hline $\begin{array}{l}\text { Pontuação da escala do } \\
\text { Feixe de Intervenção Infeção } \\
\text { do Trato Urinário x sexo }\end{array}$ & $\begin{array}{l}\text { Masculino } \\
\text { Feminino }\end{array}$ & $\begin{array}{l}12 \\
32\end{array}$ & $\begin{array}{l}7,17 \pm 2,125 \\
8,84 \pm 2,516\end{array}$ & $t=-2,048$ & 42 & $<0,047$ \\
\hline $\begin{array}{l}\text { Pontuação da escala do } \\
\text { Feixe de Intervenção Infeção } \\
\text { do Trato Urinário x Grupo } \\
\text { etário }\end{array}$ & $\begin{array}{l}26-37 \text { anos } \\
38-50 \text { anos }\end{array}$ & $\begin{array}{l}18 \\
25\end{array}$ & $\begin{array}{l}8,67 \pm 2,473 \\
8,12 \pm 2,587\end{array}$ & $t=0,696$ & 41 & $\geq 0,490$ \\
\hline $\begin{array}{l}\text { Pontuação da escala do } \\
\text { Feixe de Intervenção Cateter } \\
\text { Vascular Central x sexo }\end{array}$ & $\begin{array}{l}\text { Masculino } \\
\text { Feminino }\end{array}$ & $\begin{array}{l}12 \\
32\end{array}$ & $\begin{array}{l}13,25 \pm 4,003 \\
16,41 \pm 5,303\end{array}$ & $t=1,867$ & 42 & $\geq 0,069$ \\
\hline $\begin{array}{l}\text { Pontuação da escala do } \\
\text { Feixe de Intervenção Cateter } \\
\text { Vascular Central x Grupo } \\
\text { etário }\end{array}$ & $\begin{array}{l}26-37 \text { anos } \\
38-50 \text { anos }\end{array}$ & $\begin{array}{l}18 \\
25\end{array}$ & $\begin{array}{l}16,56 \pm 5,793 \\
14,92 \pm 4,699\end{array}$ & $\mathrm{t}=1,021$ & 41 & $\geq 0,313$ \\
\hline $\begin{array}{l}\text { Pontuação da escala do } \\
\text { Feixe de Intervenção } \\
\text { Pneumonia Associada à } \\
\text { Intubação x sexo }\end{array}$ & $\begin{array}{l}\text { Masculino } \\
\text { Feminino }\end{array}$ & $\begin{array}{l}12 \\
32\end{array}$ & $\begin{array}{l}22,83 \pm 3,639 \\
19,69 \pm 4,525\end{array}$ & $t=2,156$ & 42 & $<0,037$ \\
\hline $\begin{array}{l}\text { Pontuação da escala do } \\
\text { Feixe de Intervenção } \\
\text { Pneumonia Associada à } \\
\text { Intubação x Grupo etário }\end{array}$ & $\begin{array}{l}26-37 \text { anos } \\
38-50 \text { anos }\end{array}$ & $\begin{array}{l}18 \\
25\end{array}$ & $\begin{array}{l}18,44 \pm 3,312 \\
22,24 \pm 4,603\end{array}$ & $\mathrm{t}=2,982$ & 41 & $<0,005$ \\
\hline
\end{tabular}

Fonte: Elaboração própria.

No que se refere à associação entre a aplicação dos FI e as variáveis de experiência profissional e formação profissional, a média da pontuação do FI da ITU não diferiu entre os enfermeiros com diferentes tempos de exercício profissional, nem entre os enfermeiros que possuíam e não possuíam formação. Entretanto, encontraram-se diferenças estatísticas significativas entre a média da pontuação deste FI dos enfermeiros com diferentes tempos de exercício profissional na UCI. Os enfermeiros com menos anos de experiência na UCI obtiveram uma média mais elevada $(9,39>7,69)$, significando que seus procedimentos eram mais adequados.
A média da pontuação do FI do CVC não divergiu entre os enfermeiros com diferentes tempos de exercício profissional, assim como entre os enfermeiros com diferentes tempos de exercício profissional na UTI e diferentes condições formativas.

O mesmo sucedeu relativamente à média da pontuação do FI da PAI, que não diferiu entre o grupo de enfermeiros que possuía e o grupo que não possuía formação. A média da pontuação do FI da PAI distinguiu-se entre os enfermeiros enquadrados nas categorias de tempo de exercício profissional; uma média mais elevada foi obtida pelos que possuíam mais experiência, isto é, o 
seu procedimento nesse FI era mais adequado. Igualmente aconteceu quanto à média da pontuação do FI da PAI entre os enfermeiros enquadrados nas categorias de tempo de exercício profissional na UTI. Os que detinham mais experiência também obtiveram a média mais elevada, o que quer dizer melhor procedimento neste FI (Tabela 4).

Tabela 4-Associação entre a aplicação dos Feixes de Intervenção e as variáveis experiência profissional e formação. Penafiel, Portugal - 2019. (N=44)

\begin{tabular}{|c|c|c|c|c|c|c|}
\hline Variáveis & Categorias & $\mathbf{n}$ & $\begin{array}{c}\text { Média } \pm \text { desvio } \\
\text { padrão }\end{array}$ & $\begin{array}{c}\text { Valor } \\
\text { do teste }\end{array}$ & $\begin{array}{c}\text { Graus de } \\
\text { Liberdade }\end{array}$ & $\begin{array}{l}\text { Teste t de } \\
\text { Student p }\end{array}$ \\
\hline $\begin{array}{l}\text { Pontuação da escala do Feixe } \\
\text { de Intervenção Infeção do } \\
\text { Trato Urinário x tempo de } \\
\text { exercício profissional }\end{array}$ & $\begin{array}{c}\text { 3-15 anos } \\
16-30 \text { anos }\end{array}$ & $\begin{array}{l}17 \\
27\end{array}$ & $\begin{array}{l}8,53 \pm 2,401 \\
8,30 \pm 2,614\end{array}$ & $\mathrm{t}=0,297$ & 42 & $\geq 0,768$ \\
\hline $\begin{array}{l}\text { Pontuação da escala do Feixe } \\
\text { de Intervenção Infeção do } \\
\text { Trato Urinário x tempo de } \\
\text { exercício na Unidade de } \\
\text { Terapia Intensiva }\end{array}$ & $\begin{array}{l}\text { 1-8 anos } \\
9-20 \text { anos }\end{array}$ & $\begin{array}{l}18 \\
26\end{array}$ & $\begin{array}{l}9,39 \pm 2,146 \\
7,69 \pm 2,542\end{array}$ & $t=2,316$ & 42 & $<0,026$ \\
\hline $\begin{array}{l}\text { Pontuação da escala do Feixe } \\
\text { de Intervenção Infeção do } \\
\text { Trato Urinário x formação }\end{array}$ & $\begin{array}{l}\text { c/ formação } \\
\text { s/ formação }\end{array}$ & $\begin{array}{l}18 \\
26\end{array}$ & $\begin{array}{l}8,89 \pm 2,763 \\
8,04 \pm 2,306\end{array}$ & $t=1,404$ & 42 & $\geq 0,274$ \\
\hline $\begin{array}{l}\text { Pontuação da escala do } \\
\text { Feixe de Intervenção Cateter } \\
\text { Vascular Central x tempo de } \\
\text { exercício profissional }\end{array}$ & $\begin{array}{l}\text { 3-15 anos } \\
16-30 \text { anos }\end{array}$ & $\begin{array}{l}17 \\
27\end{array}$ & $\begin{array}{l}17,35 \pm 5,533 \\
14,41 \pm 4,618\end{array}$ & $\mathrm{t}=1,908$ & 42 & $\geq 0,063$ \\
\hline $\begin{array}{l}\text { Pontuação da escala do } \\
\text { Feixe de Intervenção Cateter } \\
\text { Vascular Central x tempo de } \\
\text { exercício na UTI }\end{array}$ & $\begin{array}{l}\text { 1-8 anos } \\
9-20 \text { anos }\end{array}$ & $\begin{array}{l}18 \\
26\end{array}$ & $\begin{array}{l}16,50 \pm 4,033 \\
14,88 \pm 5,764\end{array}$ & $t=1,026$ & 42 & $\geq 0,311$ \\
\hline $\begin{array}{l}\text { Pontuação da escala do } \\
\text { Feixe de Intervenção Cateter } \\
\text { Vascular Central x formação }\end{array}$ & $\begin{array}{l}\text { c/ formação } \\
\text { s/ formação }\end{array}$ & $\begin{array}{l}18 \\
26\end{array}$ & $\begin{array}{l}14,56 \pm 5,249 \\
16,23 \pm 5,046\end{array}$ & $t=-1,065$ & 42 & $\geq 0,293$ \\
\hline $\begin{array}{l}\text { Pontuação da escala do Feixe } \\
\text { de Intervenção Pneumonia } \\
\text { Associada à Intubação x tempo } \\
\text { de exercício profissional }\end{array}$ & $\begin{array}{l}\text { 3-15 anos } \\
16-30 \text { anos }\end{array}$ & $\begin{array}{l}17 \\
27\end{array}$ & $\begin{array}{l}17,82 \pm 3,167 \\
22,26 \pm 4,390\end{array}$ & $t=-3,610$ & 42 & $<0,001$ \\
\hline $\begin{array}{l}\text { Pontuação da escala do Feixe } \\
\text { de Intervenção Pneumonia } \\
\text { Associada à Intubação x } \\
\text { tempo de exercício na } \\
\text { Unidade de Terapia Intensiva }\end{array}$ & $\begin{array}{l}\text { 1-8 anos } \\
9-20 \text { anos }\end{array}$ & $\begin{array}{l}18 \\
26\end{array}$ & $\begin{array}{l}18,61 \pm 3,071 \\
21,88 \pm 4,869\end{array}$ & $t=-2,732$ & 41,72 & $<0,009$ \\
\hline $\begin{array}{l}\text { Pontuação da escala do Feixe } \\
\text { de Intervenção Pneumonia } \\
\text { Associada à Intubação x } \\
\text { formação }\end{array}$ & $\begin{array}{l}\text { c/ formação } \\
\text { s/ formação }\end{array}$ & $\begin{array}{l}17 \\
27\end{array}$ & $\begin{array}{l}20,28 \pm 5,062 \\
20,73 \pm 4,143\end{array}$ & $t=0,326$ & 42 & $\geq 0,746$ \\
\hline
\end{tabular}

Fonte: Elaboração própria.

Relativamente à associação entre a aplicação dos FI e os procedimentos preventivos, a média de ordenação do FI da ITU não diferiu significativamente entre os enfermeiros com diferentes procedimentos de utilização de luvas (KW:p $\geq 0,064)$.
O mesmo sucedeu no caso do FI do CVC, no qual a média de ordenação não divergiu significativamente entre os enfermeiros com diferentes procedimentos de utilização de luvas (KW:p $\geq 0,294)$. No FI da PAI, a média de ordenação da pontuação desse feixe dissentiu 
significativamente entre os enfermeiros com diferentes procedimentos de utilização de luvas (KW:p<0,022). Os enfermeiros que assinalaram utilizar sempre as luvas obtiveram a média de ordenação mais elevada $(26,50>14,71)$, significando terem procedimentos mais adequados nesse âmbito.

No que diz respeito à associação entre a pontuação dos FI e os procedimentos de auditoria, no FI da ITU, a média de ordenação da pontuação não diferiu significativamente entre os enfermeiros com distintas condições de realização das auditorias (Sim/Não) (MW:p $\geq 0,519$ ). O mesmo aconteceu no caso do FI do CVC, no qual a média de ordenação da pontuação também não divergiu significativamente entre os enfermeiros com diferentes condições de realização das referidas auditorias (MW:p $\geq 0,345$ ), assim como no caso do FI da PAI, no qual não se constataram diferenças estatísticas significativas entre a média de ordenação da pontuação dos enfermeiros com diferentes procedimentos de aplicação das auditorias (MW:p $\geq 0,232$ ).

\section{Discussão}

A maioria da amostra de enfermeiros era composta por profissionais do sexo feminino. Este resultado está em consonância com os dados estatísticos publicados pela organização representativa dos enfermeiros em Portugal ${ }^{(18)}$, nos quais se constata que, em 2018, cerca de $82,2 \%$ dos membros inscritos eram mulheres. A representatividade do sexo feminino é muito semelhante à encontrada num estudo realizado em Bragança (Portugal) ${ }^{(19)}$, com uma amostra 20 enfermeiros, no qual o objetivo era identificar os procedimentos de enfermagem em doentes submetidos a ventilação mecânica invasiva e o desenvolvimento de pneumonia, num serviço de medicina intensiva, em que a maioria da amostra pertencia ao sexo feminino (75\%).

No que se refere ao grupo etário, a maior parte da amostra enquadrava-se no intervalo dos 38-50 anos, estando este resultado de acordo com os dados publicados pela organização representativa dos enfermeiros em Portugal ${ }^{(18)}$, nos quais o grupo etário entre 36 e 50 anos representava a maioria dos enfermeiros inscritos nessa organização profissional (35,7\%), ressalvando-se o menor percentual e a não total correspondência dos grupos etários.

Quanto às habilitações académicas, neste estudo, a maioria da amostra possuía licenciatura, o que vai ao encontro dos dados publicados pela organização profissional $^{(18)}$, em que o maior número dos enfermeiros inscritos possuía a licenciatura $(74,8 \%)$, e dos resultados obtidos no estudo de Bragança (PT) ${ }^{(19)}$, no qual o maior grupo (30\%) também possuía a licenciatura.

O facto de a maioria dos enfermeiros da amostra enquadrar-se no intervalo dos 16-30 anos de experiência profissional $(61,4 \%)$ e entre os 9-20 anos de experiência profissional na UTI $(59,1 \%)$ indica que são profissionais com muita experiência profissional, o que é essencial para uma boa prática de cuidados ${ }^{(20)}$.

Mais da metade da amostra fez formação (Pós-Graduações e Especializações), o que demonstra a preocupação dos enfermeiros na procura de formação profissional. Isto significa que o próprio contexto de trabalho é rico em experiências de formação contínua, gerador de oportunidades de aprender em conjunto, com os outros, contribuindo para a formação de estudantes e profissionais e, por último, para a qualidade dos cuidados de saúde ${ }^{(21)}$.

Do grupo de enfermeiros que fizeram formação pós-graduada, metade possuía a especialidade médico-cirúrgica. Este resultado corrobora o obtido em estudo ${ }^{(19)}$, no qual o maior grupo (15\%) possuía a especialidade em Enfermagem Médico-cirúrgica, o que era expectável, uma vez que se tratou de estudo realizado em contexto de UTI.

A maioria dos profissionais não possuía formação na área de Gestão de Risco, Segurança e Prevenção de Infeção (56,8\%). Entre os que detinham esta formação, a maior parte não abordou o tema FI $(63,1 \%)$ e referiu que sentia a necessidade de abordá-lo (80,0\%). Esta necessidade é compreensível, se levar-se em conta o que refere a organização representativa dos enfermeiros em Portugal $^{(22)}$, ao dizer que a necessidade de 
formação resulta da experiência individual de cada vivência, relativamente ao processo de formação.

O Programa de Prevenção e Controlo de Infeções e de Resistência aos Antimicrobianos ${ }^{(10)}$ indica que, em 2016, a monitorização de uso de luvas pelos profissionais de saúde obteve um índice global de qualidade de $80,7 \%$, percentual bastante mais elevado do que o do presente estudo, significando que ainda há necessidade de insistir para que este comportamento consolide-se na prática desses enfermeiros.

No que diz respeito às auditorias, estudo realizado em Minas Gerais (Brasil) ${ }^{(23)}$, acerca da avaliação dos registros de enfermagem em hemoterapia de um hospital geral, revelou que apenas $65,5 \%$ dos itens avaliados nas prescrições estava em conformidade, percentual um pouco superior à execução da monitorização da adesão aos FI no presente estudo, o que expõe a diferença entre as orientações técnicas e o que é, na realidade, implementado.

No presente estudo, existe associação entre a aplicação do FI da PAI e o tempo de exercício profissional e o tempo de exercício na UTI. Nestes dois casos, os enfermeiros com maior experiência profissional (16-20 e 9-20 anos de experiência) foram os que possuíam procedimentos mais adequados neste âmbito. Estes resultados poderão ser explicados recorrendo-se às afirmações de uma autora ${ }^{(20)}$, que refere que mais tempo de exercício profissional demonstra mais competências dos enfermeiros na respetiva área de prestação de cuidados. No entanto, observa-se uma exceção a esta tendência na associação entre a aplicação do FI da ITU e o tempo de exercício profissional, na qual foram os enfermeiros com menor tempo de exercício profissional na UTI (1-8 anos) que reportaram possuir procedimentos mais adequados. Este resultado poderá ficar a dever-se ao facto de, durante a graduação, ser aprofundado o tema ITU em vários contextos, sendo uma medida de infeção mais utilizada, estando, assim, mais presente na sua curta experiência profissional.

Também se constatou existir associação entre a aplicação do FI da PAI e o uso de luvas pelos enfermeiros, enquanto procedimento preventivo de infeções, na qual os enfermeiros que assinalaram usar luvas reportaram procedimentos mais adequados. Isto poderá ser conhecido nas recomendações da DGS ${ }^{(24)}$ de utilização de luvas como procedimento preventivo.

As principais limitações deste estudo prendem-se ao facto de ser uma amostra não aleatória, o que restringiu a segurança da inferência dos resultados para a população, uma vez que, por se tratar de uma amostragem de conveniência, afetou a representatividade da amostra na UCIC.

Este estudo poderá ter implicações na prática profissional, na área da Enfermagem da Pessoa em Situação Crítica, uma vez que a sua realização e a divulgação dos resultados irão sensibilizar os profissionais de Enfermagem para a importância da adoção dessas medidas e para a necessidade de formação contínua no âmbito da prevenção e do controlo das infeções. Já está a ser perspetivada, a curto prazo, a organização de uma formação nos diferentes serviços, onde se realizou o estudo e em outros serviços do mesmo centro hospitalar, sobre esta temática, na qual pretende-se ser os dinamizadores. A mais-valia deste estudo foi ter identificado fatores associados com a aplicação de FI em UTI, o que constituiu algo inovador nesta área geográfica e nesta instituição, tendo sido propostas medidas para mitigar as infeções em estudo.

\section{Conclusão}

A análise dos resultados e da sua discussão permite identificar, quanto à associação entre a aplicação de FI pelos enfermeiros de uma UTI portuguesa e as caraterísticas sociodemográficas, no que concerne à aplicação do FI da ITU, o fator sexo e a aplicação do FI da PAI, o fator sexo e grupo etário. Quando a associação em causa, entre a aplicação dos FI e a experiência profissional e a formação os fatores emergentes, foram, no caso do FI da ITU, o tempo de exercício profissional na UTI e, no que se refere ao FI da PAI, o tempo de exercício profissional global e na UTI. Por último, no que se refere à associação entre os FI e os procedimentos preventivos, o 
único fator identificado foi a utilização de luvas, relativamente ao FI da PAI. Salienta-se o facto de, na aplicação do FI do CVC, não ter sido identificado qualquer fator associado com esta infeção.

Pode-se concluir que a formação na área de Gestão de Risco, Segurança e Prevenção de Infeção atinge menos de metade da amostra e terá que ser mais oferecida pelas entidades responsáveis, para que possa abranger a totalidade dos profissionais.

A utilização de luvas na prestação de cuidados de enfermagem, nos procedimentos em que é recomendado, poderá ainda não estar a ser cumprida por todos os profissionais, o que poderá constituir um risco de propagação de IACS. Tendo em conta que as auditorias aos procedimentos de aplicação dos FI da ITU, CVC e PAI não são uma realidade generalizada e a sua frequência é reduzida, pode-se supor que a generalização desse processo a todos os FI, assim como o aumento da sua frequência, poderá constituir medidas a aplicar para minimizar aquela lacuna e até para aumentar a aplicação do FI, garantindo-se a qualidade dos procedimentos.

Assim, a implementação dessas estratégias para as organizações de saúde é urgente. Este objetivo a alcançar depende não só das diretivas e dos sistemas de auditorias que fazem parte da estrutura organizacional, mas, sobretudo, dos profissionais de saúde que têm a responsabilidade do uso de boas práticas, podendo o cidadão beneficiar-se de cuidados de qualidade.

\section{Fontes de financiamento}

Este estudo teve o financiamento dos seguintes Centros de Investigação: Centro de Investigação em Estudos da Criança (CIEC), pelo Projeto Estratégico UID/CED/00317/2013, por meio dos Fundos Nacionais da Fundação para a Ciência e a Tecnologia (FCT), cofinanciado pelo Fundo Europeu de Desenvolvimento Regional (FEDER) por meio do COMPETE 2020 - Programa Operacional Competitividade e Internacionalização (POCI) com a referência POCI-01-0145-FEDER-007562, Braga, Portugal; Health Sciences Research Unit: Nursing
(UICISA: E), pelo projeto Estratégico UIDB/00742/2020, por meio dos Fundos Nacionais da Fundação para a Ciência e a Tecnologia (FCT), Nursing School of Coimbra (ESEnfC), Portugal.

\section{Colaborações:}

1 - concepção, projeto, análise e interpretação dos dados: Ana Gertrudes Felgueiras Machado, Amâncio António de Sousa Carvalho e Ana Paula Morais Carvalho Macedo;

2 - redação do artigo e revisão crítica relevante do conteúdo intelectual: Ana Gertrudes Felgueiras Machado, Amâncio António de Sousa Carvalho e Ana Paula Morais Carvalho Macedo;

3 - aprovação final da versão a ser publicada: Ana Gertrudes Felgueiras Machado, Amâncio António de Sousa Carvalho e Ana Paula Morais Carvalho Macedo.

\section{Referências}

1. Portugal. Ministério da Saúde. Despacho n. 9 5613/2015. Aprova a Estratégia Nacional para a Qualidade na Saúde 2015-2020 [Internet]. Lisboa (PT); 2015. Diário da República, № 102, de 27 de maio de 2015. Série II [cited $2021 \mathrm{Feb} 9]$. Available from: https://dre.pt/web/guest/pesquisa/ -/search/67324029/details/normal?l=1

2. Vesco NL, Fragoso LVC, Beserra FM, Aguiar MIF, Alves NP, Bonates LAM. Healthcare-related infections and factors associated to the postoperative period of liver transplantation. Texto Contexto Enferm. 2018;27(3):e:2150017. DOI: http://dx.doi.org/10.1590/0104-0707201800 02150017

3. Curan GRF, Rossetto EG. Interventions to decrease catheter-associated bloodstream infections in newborns: an integrative review. Texto Contexto Enferm. 2017;26(1):e:5130015. DOI: http://dx.doi.org/10.1590/0104-0707201700 5130015

4. Silva AG, Oliveira AC. Impact of the bundles implementation on the reduction of bloodstream infections: an integrative review. Texto Contexto Enferm. 2018;27(1):e3540016. DOI: http://dx.doi.org/10.1590/0104-07072018003 540016 
5. Danski MTR, Pontes L, Schwanke AA, Lind J. Infecção da corrente sanguínea relacionada a cateter venoso central para hemodiálise: revisão integrativa. Rev baiana enferm. 2017;31(1):e16342. DOI: $10.18471 /$ rbe.v31i1.16342

6. Danski MTR, Pedrolo E, Boostel R, Souza A, Felix JVC. Custos da infecção relacionada a cateter venoso central em adultos: revisão integrativa. Rev baiana enferm. 2017;31(3):e18394 DOI: $10.18471 /$ rbe.v31i3.18394

7. Lopes M, Zanchett CCC. Urinary tract infections: a review on the scientific evidence of main medicinal plants used in clinical practice. FEMINA [Internet]. 2019 [cited 2021 Feb 9];47(11):824-30. Available from: https:// docs.bvsalud.org/biblioref/2019/12/1046555/ femina-2019-4711-824-830.pdf

8. Andrade VLF, Fernandes FAV. Prevention of catheter-associated urinary tract infection: implementation strategies of international guidelines. Rev Latino-Am Enfermagem. 2016;24: e2678. DOI: http://dx.doi.org/10.1590/1518-8345. 0963.2678

9. Alkhawaja S, Alkhawaja S, Saeed NK, Fawzy N, El A, Azam M, et al. Catheter-Associated Urinary Tract Infections at Intensive Care Unit in Bahrain. EC Microbiology [Internet]. 2017 [cited 2021 Feb 9];8(2):71-9. Available from: https://www. researchgate.net/publication/319037391_EC_ MICROBIOLOGY_Research_Article_CatheterAssociated_Urinary_Tract_Infections_at_Intensive_ Care_Unit_in_Bahrain

10. Portugal. Ministério da Saúde, Direção-Geral da Saúde. Programa de Prevenção e Controlo de Infeções e de Resistência aos Antimicrobianos 2017 [Internet]. Lisboa (PT); 2017 [cited 2021 Feb 9]. Available from: https://www.sns.gov.pt/ wp-content/uploads/2017/12/DGS_PCIRA_V8.pdf

11. Reis AFGME. Prevenção da Pneumonia Associada à Ventilação na Pessoa em Situação Crítica - intervenção especializada de enfermagem [Relatório de Estágio]. Lisboa (PT): Escola Superior de Enfermagem de Lisboa; 2017.

12. European Centre for Disease Prevention and Control. Healthcare-associated infections in intensive care units - Annual Epidemiological Report for 2017, from 1 January 2017 until 31 December 2017 [Internet]. Stockholm; 2019 [cited 2021 Feb 9] Available from: https://www. ecdc.europa.eu/en/publications-data/healthcare- associated-infections-intensive-care-units-annualepidemiological-1

13. Gao F, Wu Y-Y, Zou J-N, Zhu M, Zhang J, Huang $\mathrm{H}-\mathrm{Y}$, et al. Impact of a bundle on prevention and control of healthcare associated infections in intensive care unit. J Huazhong Univ Sci Technolog Med Sci. 2015;35(2):283-90. DOI: https://pubmed.ncbi.nlm.nih.gov/25877366/

14. McAlearney AS, Hefner JL, Sieck CJ, Walker DM, Aldrich AM, Sova LN, et al. Searching for management approaches to reduce HAI transmission (SMART): a study protocol. Implement Sci. 2017;12(82):1-11. DOI: DOI 10.1186/s13012-017-0610-Z

15. Portugal. Ministério da Saúde, Direção-Geral da Saúde. Orientação no 002/2017, de 20 de janeiro de 2017. Preparação e Condução de Auditorias da Qualidade e Segurança da Prestação de Cuidados de Saúde [Internet]. Lisboa (PT); 2017. [cited 2021 Feb 9]. Available from: https://nocs.pt/preparacaoe-conducao-de-auditorias-da-qualidade-eseguranca-da-prestacao-de-cuidados-de-saude/

16. Vilelas J. Investigação. O processo de construção do conhecimento. $3 \mathrm{a}$ ed. Lisboa (PT): Edições Sílabo; 2020.

17. Marôco J. Análise Estatística com o SPSS Statistics. 7a ed. Pêro Pinheiro (PT): Report Number; 2018.

18. Ordem dos Enfermeiros. Estatística de Enfermeiros. Anuário 2018 [Internet]. Lisboa (PT); 2018 [cited 2021 Feb 9]. Available from: https:// www.ordemenfermeiros.pt/sala-de-imprensa/ estat\%C3\%ADstica-de-enfermeiros/

19. Cruz JRM, Martins MDS. Pneumonia associated with invasive mechanical ventilation: nursing care. Rev Enf Ref. 2019;IV(20):87-96. DOI: https:// doi.org/10.12707/RIV18035

20. Benner P. De iniciado a perito: excelência e poder na prática clínica de enfermagem. 2a ed. Coimbra (PT): Quarteto; 2005.

21. Macedo APMC, Padilha KG, Püschel VAA. Professional practices of education/training of nurses in an intensive care unit. Rev Bras Enferm. 2019;72(2):338-45. DOI; https://doi. org/10.1590/0034-7167-2017-0793

22. Portugal. Lei $\mathrm{n}^{\mathrm{o}} 156$, de 16 de setembro de 2015. Segunda alteração ao Estatuto da Ordem dos Enfermeiros, conformando-o com a Lei n. - 2/2013, de 10 de janeiro, que estabelece o regime jurídico de criação, organização e 
funcionamento das associações públicas profissionais [Internet]. Lisboa PT; 2015. Diário da República, № 181, de 16 de setembro de 2015, Série I [cited 2021 Feb 9]. Available from: https://dre.pt/home/-/dre/70309896/details/ maximized?p_auth=eVIwl6Va

23. Santos SP, Tanaka LH, Gusmão A, Abreu RGS, Carneiro IA, Carmagnani MIS. Evaluation of nursing records on blood transfusion in a general hospital. Av Enferm [Internet]. 2013 [cited 2021 Feb 9];31(1):103-12. Available from: http://www. scielo.org.co/pdf/aven/v31n1/v31n1a10.pdf
24. Portugal. Ministério da Saúde, Direção-Geral da Saúde. Norma no 013, de 25 de agosto de 2014 atualizada a 07/08/2015. Uso e Gestão de Luvas nas Unidades de Saúde [Internet]. Lisboa (PT); 2015 [cited 2021 Feb 9]. Available from: https://www. dgs.pt/directrizes-da-dgs/normas-e-circularesnormativas/norma-n-0132014-de-25082014.aspx

Recebido: 11 de março de 2021

Aprovado: 10 de agosto de 2021

Publicado: 15 de setembro de 2021

A Revista Baiana de Enfermagem utiliza a Licença Creative Commons - Atribuição-NãoComercial 4.0 Internacional.

https://creativecommons.org/licenses/by-nc/4.0/

Este artigo é de acesso aberto distribuído sob os termos da Licença Creative Commons (CC BY-NC).

Esta licença permite que outros remixem, adaptem e criem a partir do seu trabalho para fins não comerciais. Embora os novos trabalhos tenham de lhe atribuir o devido crédito e não possam ser usados para fins comerciais, os usuários não têm de licenciar esses trabalhos derivados sob os mesmos termos. 